\title{
GPS: User Position Calculation Including Advanced Troposphere Delay Modeling
}

\author{
Swathi Nalla
}

\begin{abstract}
Objective of this project is to analyze and mitigate troposphere delays induced in GPS signals, which can result in very large position errors while estimating user position. The standard models currently present do not take into account all the various set of parameters or elements of the troposphere that can cause a significant delay. This project also includes study of troposphere propagation delays that improve the understanding of GPS signal propagation through the troposphere during irregular conditions. This characteristic is very important as it can play crucial role in real time surveying, navigation, precision farming and positioning for emergency services. Due to the tropical nature of the Indian climate the troposphere delay can be observed significantly in India sub-continent. In order to accurately estimate delay troposphere in real time conditions is taken into account, which are provided by the Indian meteorological department, by their automatic weather surveillance systems. GPS data for stations in India is obtained from CORS data for Bangalore, from where we obtain the observation and navigation files used in the calculations. Obtained data is processed and run through various algorithms like least squares satellite position calculation, error mitigation and ray tracing algorithms to mitigate troposphere and better estimate user position.

Apart from these algorithms this project also includes a study on various concepts/formulas that help in using the forecasted real time data to be used in snell's law to estimate delay as part of ray tracing techniques. All the code development in this project is done using MATLAB by math works and GUI is developed for an easier interface. For analysis purposes the data is analyzed with and without the advanced mitigation techniques to show the improvement in position estimation using advanced troposphere mitigation techniques.
\end{abstract}

Keywords-Troposphere, Delay mitigation, ray tracing algorithm, GPS.

\section{INTRODUCTION}

GPS is a satellite-based system that was deployed by U.S. Department of Defense and is now used for positioning and navigation purposes throughout the earth. It is very accurate in providing 3-dimensional position, velocity and time information. It is comprised of 24 satellites that circle the earth precisely twice a day and send signal information to earth. GPS receivers utilize this information to calculate the exact position of the user with the help of multi-lateration techniques. A GPS system consists of three segments: the space segment, the control segment and the user segment. GPS satellite uses L-band to transmit signals to earth. The GPS signal structure consists of three parts, which comprise

Revised Manuscript Received on 14 August, 2019.

Swathi Nalla, Asst. Professor, Dept. of ECE, Malla Reddy Engineering College for Women(Autonomous), Secunderabad-TS, India swathinalla@gmail.com

of a pseudo random code, ephemeris data and almanac data. All the satellites transmit on the same frequency but to differentiate between signals from different satellites, pseudo random code is used. Ephemeris data consists of orbital information of the satellite and almanac consists of satellite's status and health information .

When the satellite signal consisting of all this information travels through the space, various factors change the speed of propagation of signals, which in turn changes the signal transit time. Transit time is the basic measurement in GPS applications. Accurate transit time multiplied by speed of light gives the distance between the user antenna and satellite that is used in multi-lateration techniques to calculate position. So even a small error in transit time accounts for loss of accuracy. Amongst those various factors that introduce delay in GPS signals, atmospheric delay is one and it consist of delays due to both ionosphere and troposphere.

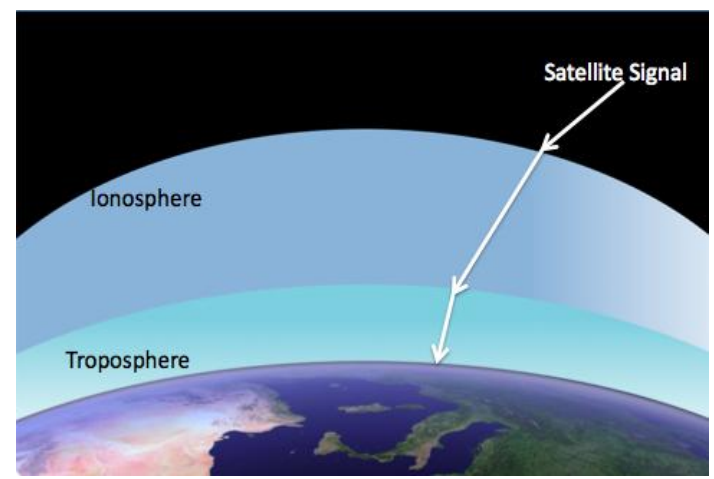

Figure 0.1 Refraction of GPS signals in atmosphere

Ionosphere is dispersive wherein the errors due to ionosphere can be modeled using two GPS signal frequencies where as on the other hand, troposphere is non dispersive hence the troposphere errors cannot be modeled using the GPS signals. Non-dispersive characteristic of the troposphere has driven many researchers to develop various models to estimate troposphere delay. These models stand good only in standard atmospheric conditions and hence cannot be relied on completely during severe weather conditions. This basic idea is the motivation behind this thesis i.e., to model troposphere delay induced in GPS signals in severe weather conditions using 3D near real time weather radar reflectivity data. In this thesis, a method of troposphere propagation delay modeling of GPS signals is suggested that uses a ray tracing method in combination with weather radar reflectivity data, to model the troposphere delays in non standard conditions i.e. large cloud, heavy rain etc. 


\section{AIM OF THE PROJECT}

Objective of this project is to analyze and mitigate troposphere delays induced in GPS signals, which can result in very large position errors while estimating user position. The standard models currently present do not take into account all the various set of parameters or elements of the troposphere that can cause a significant delay. This project also includes study of troposphere propagation delays that improve the understanding of GPS signal propagation through the troposphere during irregular conditions. This characteristic is very important as it can play crucial role in real time surveying, navigation, precision farming and positioning for emergency services. Due to the tropical nature of the Indian climate the troposphere delay can be observed significantly in India sub-continent. In order to accurately estimate delay troposphere in real time conditions is taken into account, which are provided by the Indian meteorological department, by their automatic weather surveillance systems. GPS data for stations in India is obtained from CORS data for Bangalore, from where we obtain the observation and navigation files used in the calculations. Obtained data is processed and run through various algorithms like least squares satellite position calculation, error mitigation and ray tracing algorithms to mitigate troposphere and better estimate user position.

Apart from these algorithms this project also includes a study on various concepts/formulas that help in using the forecasted real time data to be used in snell's law to estimate delay as part of ray tracing techniques. All the code development in this project is done using MATLAB by math works and GUI is developed for an easier interface. For analysis purposes the data is analyzed with and without the advanced mitigation techniques to show the improvement in position estimation using advanced troposphere mitigation techniques.

\section{METHODOLOGY}

Many studies have shown that troposphere delay is large when the parcel of air traversed by radio waves, consists of severe weather fronts and heavy rain. It is shown that troposphere zenith delay can increase up to $8 \mathrm{~cm}$ when warm weather front approaches the receiver. For various satellites at elevation angles between 5 and 90 degrees, a troposphere delay difference of up to $30 \mathrm{~cm}$ can be observed over a $5 \mathrm{~km}$ baseline. Zenith delays of up to $4.4 \mathrm{~cm}$ have been observed over a $3 \mathrm{~km}$ baseline. For a longer baseline of approximately $16 \mathrm{~km}$, up to $40 \mathrm{~cm}$ of Tropospheric delay differences for satellites above 12 degrees of elevation angles have been observed. The delay estimated in zenith direction changes and grows more prominent at low elevation angles. One of the major models that are used to mitigate troposphere delay is modified Hopfield mode and it has been shown to accurately and appropriately calculate both the dry and wet Tropospheric delays. A global 1-year data collection from June 1999 to March 2000 at 100 different sites shows that the global root mean square residual for the MHM hydrostatic zenith component is a $1 \mathrm{~cm}$, while the rms residual for the MHM wet zenith component is $3 \mathrm{~cm}$. These results are relative to a total zenith delay of approx $2.5 \mathrm{~m}$, which shows that the absolute MHM errors are approx $1 \%$ of the total delay. However MHM does not account for dispersive scattering delay due to propagation through hail, rain or snow (hydrometeors).

Another study that shows the significance of various Tropospheric components on GPS signal accuracy. This study shows that water vapor, cloud liquid, rain and sandstorms can induce significant delays and a very good description of how large of a Tropospheric error can be expected associated with each is defined. According to the comparison the delay induced due to water vapor is large compared to various other atmospheric components and can go up to $140 \mathrm{~mm} / \mathrm{km}$ for vapor diameter less than $10-7 \mathrm{~mm}$. On the other hand, cloud can induce a surface delay less than $8 \mathrm{~mm} / \mathrm{km}$ and drizzle and steady rain can induce surface delays $0.2 \mathrm{~mm} / \mathrm{km}$ and $2 \mathrm{~mm} / \mathrm{km}$ respectively which can account to $3 \%$ of the largest delay induced by dry air and water vapor. This study also outlines that water vapor is having significant role and occupies a large part in the wet delay.

Having outlined that, the next focus lies on the estimation of variables required to calculate the wet refractivity from the available data i.e. the three-dimensional radar reflectivity returns in the case of this thesis. On a general top-level view, we require liquid water content to calculate refractivity associated with clouds, rain rate to calculate the refractivity associated with rain and relative humidity information to calculate the refractivity associated with water vapor. Calculations related to the above variables would be discussed as part of the approach . One of the challenges is to relate the radar reflectivity $\mathrm{dBz}$ values to the liquid water content. There are many papers that have outlined the equations to empirically model the relation. However the drawback in these studies is that it is only valid at certain regions of the curve fitting and would not give reasonable results. All the studies described above helps in supporting the idea of using near real time weather parameters to model, varying wet delays. This approach has potential to estimate the troposphere delay very closely to the actual delay that can be expected in severe weather.

\section{Ray tracing algorithm for total troposphere delay calculation}

The algorithm described below closely follows appendix A3 in [4], with a change in subscripts to correct a notational problem and also a more computationally efficient way of calculating geometric bending effect is used. Only the algorithm itself is described below, while the full derivation is provided in [Appendix A]. There are some assumptions and notations in this approach that are described below. With reference to Figure 3.1, the following is noted.

1. "the ray" stands for signal transmission path from a point to the satellite.

2 . The height $h$ of a layer $i$ is the distance from the surface of the earth to the point of intersection of the ray on the line of curvature of the respective layer.

3. A piercing point $P$ stands for the point of intersection of the ray with the line of intersection between two adjoining layers (Note: Not to be confused with the total pressure notation in Section 4.1).

4. A subscript $i$ to any parameter corresponds to a layer $i$ and the numbering starts from 0 denoting a point on the ground and moving up 
towards the end of the troposphere.

5. For the purpose of this thesis, the atmosphere up to a height of $10 \mathrm{~km}$ is modeled.

6. The refractive index $n$ is assumed to remain constant throughout the layer under consideration and $n$ is calculated using the various formulae described in section 0 .

7. The step size, which is also referred to as the height of the layer, is typically 50 meters close to the surface of the earth, and becomes larger the height goes up into the atmosphere. This is due to the fact that the troposphere activity decreases as we go higher in the troposphere towards the tropopause.

8. Path length $S i$ is the distance between piercing points of adjoining layers, and outgoing elevation angle is the angle made by the outgoing ray with the tangential plane of the layer. $z i$ and $y i$ are the geocentric " $z$ " and " $\mathrm{y}$ " coordinates of the corresponding point along ray traced path. Distances $r i$ to the geo center from the piercing points $P$ at all layers are determined by adding the radius of the earth to the heights of the layers $h i$.

\section{IMPLEMENTATION}

This chapter outlines a brief summary on the troposphere delay classifications and the various methods that researchers have resorted to, in order to account for the troposphere delay. Troposphere is considered electrically neutral space that has index of refraction slightly greater than unity. This property of troposphere reduces the velocity of wave propagation when compared to the expected velocity of speed of light in unity index of refraction. As a result of this the apparent range from user to satellite gets extended by certain amount, which is typically called troposphere delay in GPS applications. Not only do the waves propagate with reduced velocity but also get slightly bent due to change in index of refraction with height and with various components present in troposphere. Various components that contribute to the refraction of signals in troposphere are dry gases, water vapor, suspended water droplets and rain. Each one of these components has different index of refraction associated with them and hence are modeled differently. However when considering the index of refraction of a parcel of air, the combined effect of index of refraction of various components is considered.

Troposphere delay can be broken down into two major types: one is dry delay contributed by dry gases and another is wet delay contributed by cloud, rain, suspended water droplets etc. Dry delay constitutes of $90 \%$ of the total troposphere delay and depends on the pressure fields that very slowly and can be modeled easily. Wet delay is $10 \%$ of the total delay and is hard to predict and model considering the fact that profile of the water vapor, clouds, and rain present in troposphere is ever changing and is hard to model accurately using standard conditions.

The simple troposphere total delay is nothing but the sum of the dry and wet delay along the signal path. Dry and wet delays can be written in the form of refractivities for convenience and hence the delay equation in [m] can be written as below where the refractivities are integrated along the signal path $l$.

$$
\text { Delay }_{\text {total }}=10^{-6} \int\left[N_{d r y}(l)+N_{\text {wet }}(l)\right] d l
$$

Dry and wet refractivity can be expressed as following equations respectively.

$$
\begin{aligned}
& N_{d r y}=77.64\left(\frac{P}{T}\right) \\
& N_{\text {wet }}=3.73 * 10^{5}\left(\frac{e}{T^{2}}\right)
\end{aligned}
$$

Where $\mathrm{P}, \mathrm{T}$ and $\mathrm{e}$ are the total pressure in $[\mathrm{mb}]$, temperature in $[\mathrm{k}]$ and $\mathrm{e}$ is the partial pressure of water vapor in $[\mathrm{mb}]$.

Due to this highly variable nature the wet delays can be as large as $50 \%$ of the dry delay in severe weather conditions. This fact provides motivation to use near real time weather radar data in models instead of standard atmosphere profiles when considering troposphere delay calculation in severe weather. With the knowledge of near real time weather, troposphere delay can be modeled with better accuracy. For simplicity index of refraction will be expressed in terms of refractivity throughout this thesis, which is expressed given as following equation.

$$
N=(n-1) * 10^{6}
$$

There are many models that estimate the troposphere delay based on location and seasonal assumptions. Two models that are vastly used in the GPS community are described below to give an insight on the troposphere models. They are called Saastamo inen and Hopfield models. These models calculate the total delay introduced in troposphere by estimating the delay induced due to average meteorological conditions, e.g. pressure, temperature and relative humidity, along the propagation path of a signal . The general estimation of troposphere delay by Saastamoinen and Hopfield models can be outlined in two steps.

1. First they estimate the total troposphere zenith delay (both the dry and wet delay component of troposphere in zenith direction).

$$
\text { Delay }{ }_{\text {zenith }}=\text { Delay }_{\text {zenith, dry }}+\text { Delay }_{\text {zenith,wet }}
$$

2. Then define a mapping function to scale zenith delays as a function of elevation angle.

$$
\text { Delay }_{\text {zenith }}=\left(\text { Delay }_{\text {zenith,dry }} * m_{d}(e l)\right)+\left(\text { Delay }_{\text {zenith,wet }} * m_{w}(e l)\right)
$$

Where $m_{d}$ and $m_{w}$ are the dry and wet mapping functions as a function of satellite elevations angle $e l$ in degrees.

Other types of Tropospheric correction models are summarized and compared with each other. All these methods function properly when neutral atmosphere is taken into consideration. However in severe weather scenarios, the use of average meteorological conditions introduces additional errors in both dry and wet delays and the total error can be $5-10 \mathrm{~cm}$.

The above mentioned models does not stand in play when the need for highly accurate positioning precision is required because the standard atmosphere does not take into consideration the changing profile of the atmosphere like the presence of rain clouds, snow etc which greatly induce delay in the signal. 
In order to overcome this problem a model for mitigating troposphere error is suggested that instead of calculating dry and wet delays using standard atmosphere conditions takes into account real time meteorological data from the weather radar as input for calculating troposphere error. And also instead of first calculating delay and then mapping it to elevation angle, it divides the whole curved signal transmission path into several layers and simultaneously calculates troposphere error at every layer taking as inputs real time profile of that layer from weather radar. It is a method of ray tracing which is much efficient than mapping zenith delays to elevation angles [4].

\section{STEPS FOR CALCULATING THE TOTAL DELAY}

The approach will be as described below:

Profiling the whole atmosphere into numerous layers of height $\mathrm{h}$. of all the layers using weather radar data.

With the knowledge of initial elevation angle, calculation of all the incremental distances between two points of different layers and also calculating outgoing elevation angles. The following figure explains ray tracing.

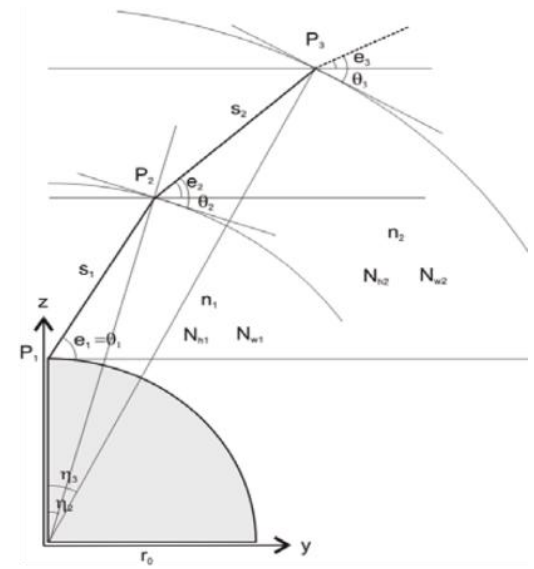

Figure 3.1 Diagram showing the ray tracing of a signal from user to satellite through troposphere

1) Then the hydrostatic and wet path delays can be calculated by the summation of incremental distances and corresponding dry and wet refractivity of each layer respectively.

2) The total delay will be the summation of dry and wet delays calculated separately for each layer. And also delay due to geometric bending is added to the hydrostatic delay. In this way we can get the accurate estimate of how much the signal got delayed through out its entire propagation path.

After this ray tracing the total delay can be calculated by the summation of wet and dry delays using the formulas.

$$
\begin{aligned}
& d_{s_{h}}=\sum_{i=1}^{k-1} s_{i} N_{h i} \\
& d_{s_{w}}=\sum_{i=1}^{k-1} s_{i} N_{w i}
\end{aligned}
$$

\section{DATA ANALYSIS AND RESULTS}

A simulated model of ray tracing has been developed and the required weather profile along the signal transmission path has been calculated using real time weather radar data from NSSL. Nearest neighbor interpolation technique is used to construct the troposphere profile along the required
Calculation of dry and wet refractivity's and refractive index

satellite transmission paths. The model calculates the index of refraction pertaining to a point along the transmission path and thus uses this in ray tracing to estimate the troposphere delay.

For the purpose of this thesis two-receiver site has been considered which are in Bangalore. The GPS data for these two stations has been obtained from CORS website. The nearest radar station to these locations is called KRAX, which is at Raleigh. For the purpose of analysis a severe weather day for these two stations has been taken into consideration, which is june 8 2011. On this particular day both the locations saw severe cloud and rainfall activity. The baseline between in that location is $\sim 5 \mathrm{~km}$. For accurate predictions the system time of weather radar is matched with the GPS time. For the purpose of differential calculations station in Bangalore is considered reference and this station is considered user. From a particular site the delay in signals from all the visible satellites is calculated using the ray-tracing model. Amongst the visible satellites, the satellite with the highest elevation angle is picked as a reference satellite for calculating double differences. The delay of each satellite at the first site is subtracted from the same satellite signal delay at second site, which forms one set of single differences. Difference in delay of reference satellites at both the site is another set of single differences. Double differences are obtained from subtracting two single differences. By subtracting single differences many common errors present are eliminated. The algorithm has been run at several instances of high troposphere activity and the GPS single and double difference for two sites is compared with the troposphere single and double differences obtained from standard modified Hopfield model. From a database of severe events only few instances are picked and are included in this summary for analysis purpose.

Following is the weather profile along the various satellite transmission paths at sites Bangalore time 5:05 on day 08/06/2011.

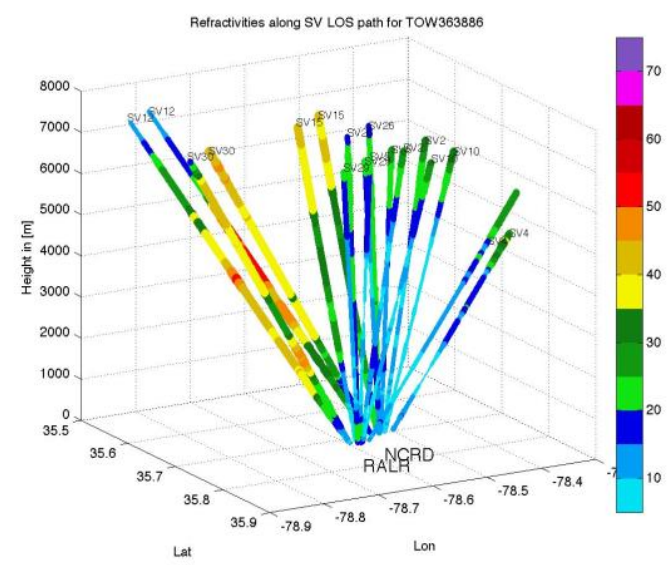

Figure 4.2 Refractivities of SV at Bangalore time 5:05 on 08/06/2011

As seen above of all the satellites, SV 12 and SV 30 observed large activity. From the amount of troposphere activity observed one can expect that the single difference delay for these satellites will be large. However, a further look into the single difference plots contradicts the expected results. 


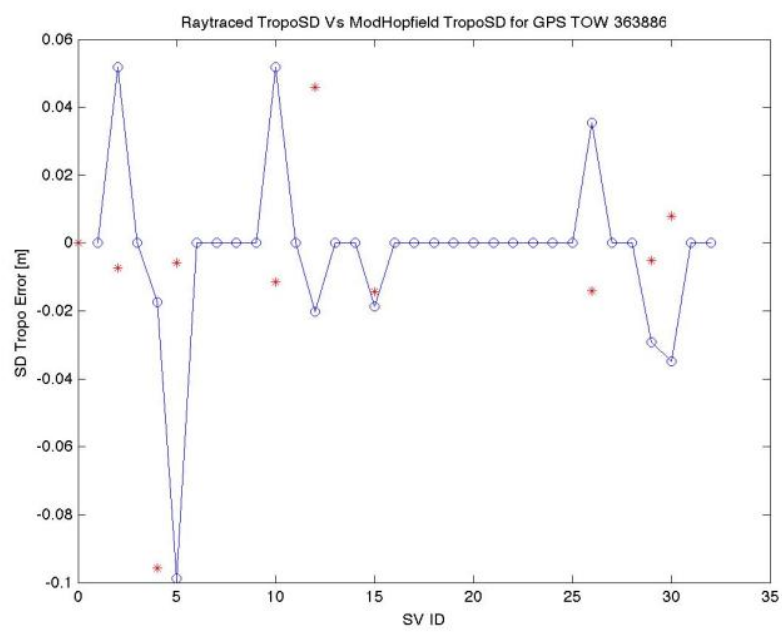

Figure 4.3 Plot of ray traced Tropospheric SD Vs standard model SD for Bangalore time 5:05

This above single difference plots reveal that the largest delay observed was with SV 5 and the delay observed by SV 12 and SV 30 are much smaller. Apart from the troposphere weather activity the other parameters that play a significant role in the magnitude of troposphere delay are the temperature and the relative humidity profiles at the two locations in consideration. These two parameters are crucial because all the other required parameters can be deduced from these two. However one of the advantage in using the weather radar data is that we get the amount of rainfall rate pertaining to certain $\mathrm{dBz}$ values seen on the radar, using which we can estimate the refractivity of rain which is shown to be a non significant value in terms of individual troposphere delay contribution. Also for the purpose of analysis a troposphere delay value less than $5 \mathrm{~cm}$ in magnitude is not considered for evaluation because that might be due to some unseen parameters. Also of all the 4 individual refractivity contributions it has been shown that the contribution of $\mathrm{N} 2$ is very significant. The information regarding the literature supporting this is included in chapter 2 . Following figure shows the refractivity values for $\mathrm{N} 1$ and $\mathrm{N} 2$ at both the locations:
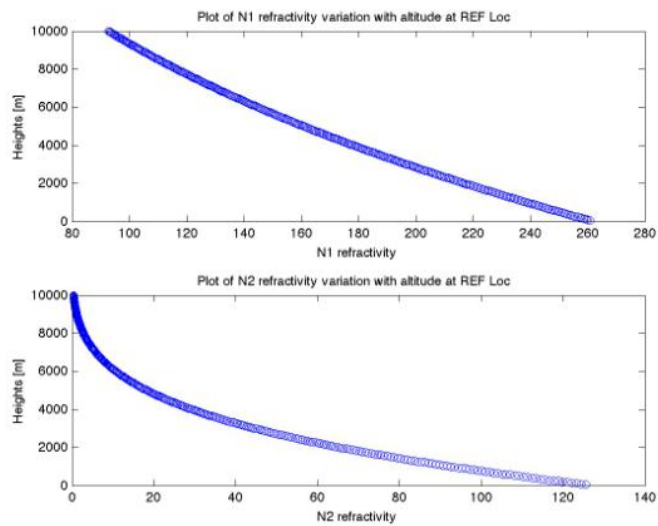

Figure4.4 Plot showing the variation of refractivity value at Bangalore location
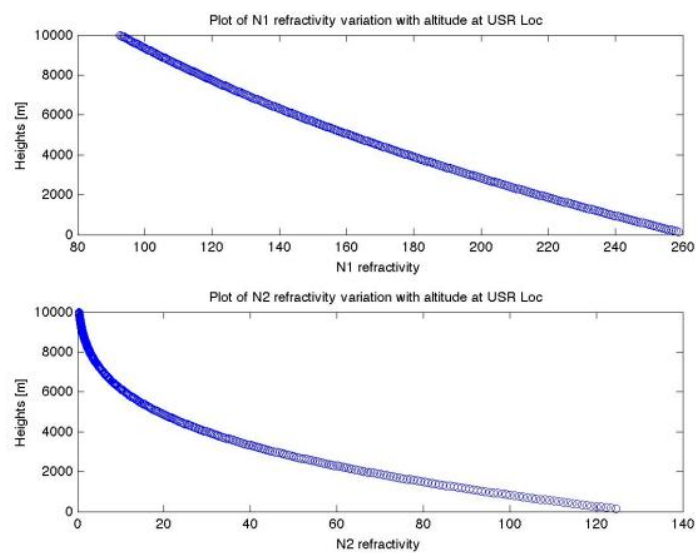

Figure 4.5 Plot showing the variation of refractivity value at Bangalore location

Plot for the next time stamp in consideration is at Bangalore time 5:20.

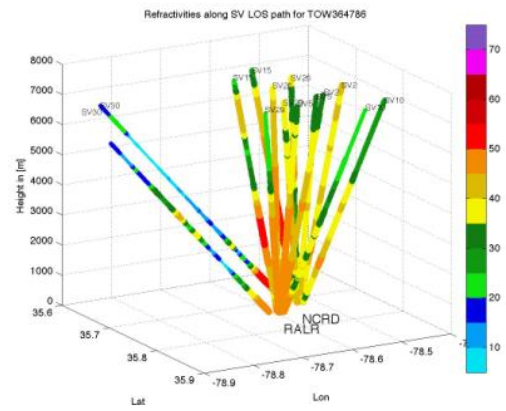

Figure4.6 Refractivities of SV at Bangalore time 5:20 on $08 / 06 / 2011$

By observing the above plot SV15 is having large activity and is expected that the delay will be large. The SD plots correlates with the weather radar plot at this time epoch.

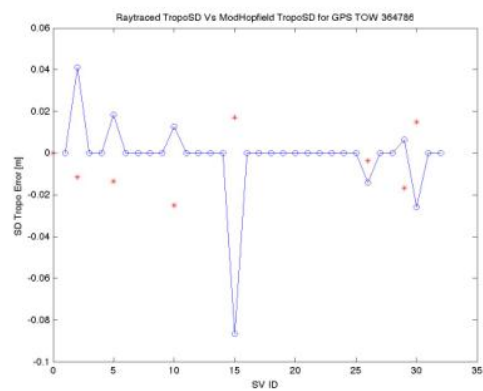

Figure4.7 Plot of ray traced Tropospheric SD Vs standard model SD for Bangalore time 5:20

Even though the other satellites see some cloud and rain activity the reflectivties observed at both the station locations are close which cancels out in the single difference calculations and hence is not reflected in the above figure. The plot of N1 and N2 at all the heights is expected to be smooth curve when there is not much of troposphere activity and shows up in the plots when the weather is severe. Following are the N1 and N2 plots at time5:20 at Bangalore . 

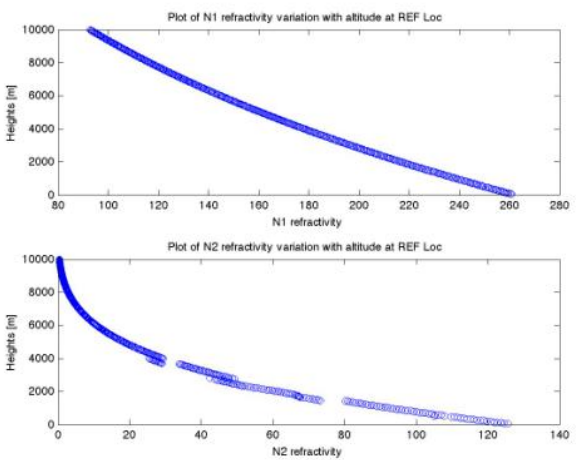

Figure4.8 Plot of variation of refractivity value at Bangalore location time 5:20
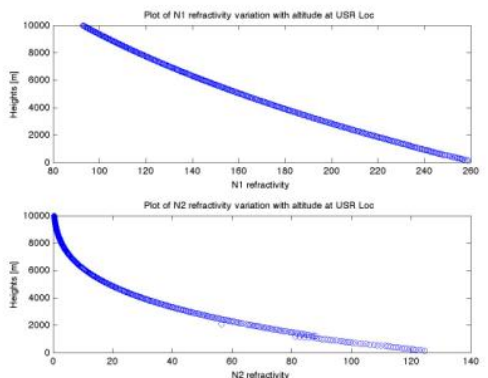

Figure4.9 Plot of variation of refractivity value at Bangalore location time 5:20

Next time stamp in consideration is at Bangalore 5:50 where both the locations saw cloud activity.

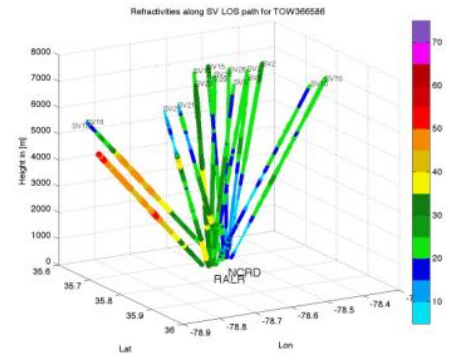

Figure4.10 Refractivities of SV at Bangalore time 5:50 on $08 / 06 / 2011$

Above weather radar data plot shows that the satellite 18 is having large troposphere activity. It should also be noted that the SV 18 is at a very low elevation angle of 11 degrees at this certain time epoch. Hence it is expected that the path traversed will be larger thus going through longer distances with possible weather activity. The weather radar correlates with the SD calculated for SV 18 at this instance, which is larger, compared to other satellites.

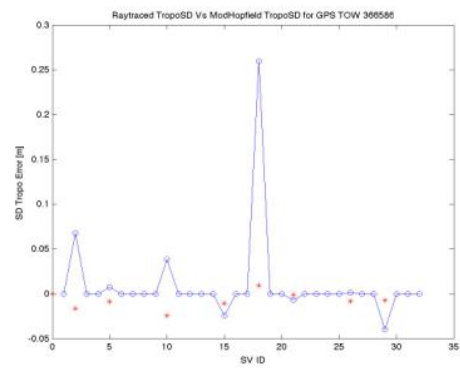

Figure4.11 Plot of ray traced Tropospheric SD Vs standard model SD for UTC time 5:50
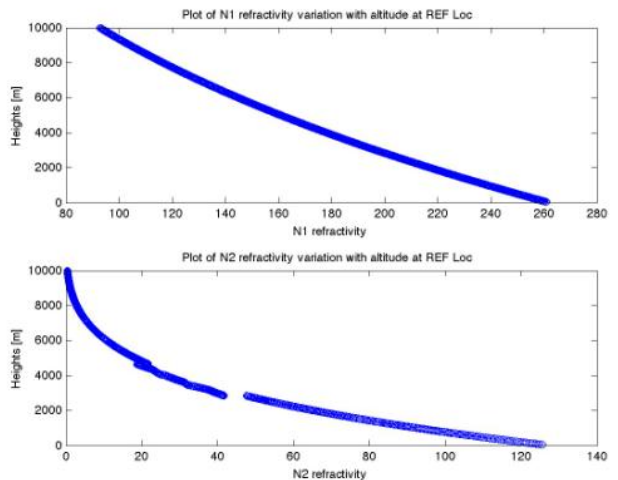

Figure4.12 Plot of variation of refractivity value at Bangalore location UTC 5:50
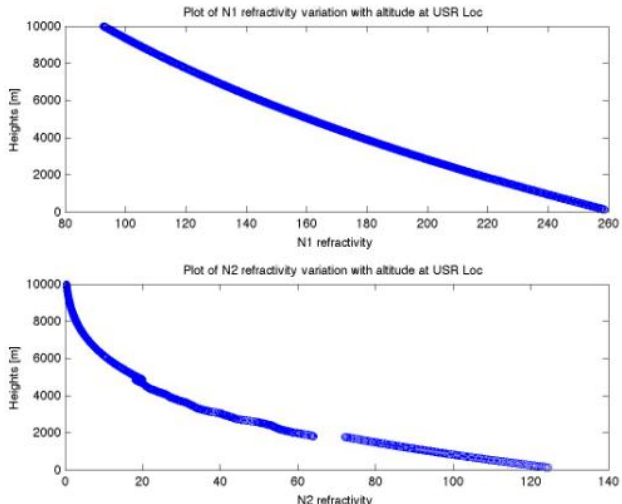

Figure4.13 Plot of variation of refractivity value at Bangalore location UTC 5:50

As seen the main parameter that plays significant role is the $\mathrm{N} 2$ refractivity. This tends to agree and disagree with the weather radar data. It is hard to correlate the weather radar reflectivity returns to the actual humidity of that location. However it can be shown that the large errors seen on the Tropospheric single differences can be correlated with the weather fronts present along the signal transmission path of the satellites.

\section{CONCLUSIONS}

From a particular site the delay in signals from all the visible satellites is calculated using the ray-tracing model. Now amongst the visible satellites from the receiver the satellite with the highest elevation angle is picked as reference satellite for calculating double differences. Now the delay of each satellite at first site is subtracted from the reference satellite delay. The same process is repeated at the second site also. Now there are two sets of satellite delays. Each set of delays at one site is nothing but satellite single differences. To get double differences the obtained single differences from site one and two are finally subtracted from each other. In this way we are eliminating common errors and this GPS double difference for two test sites is compared with the troposphere GPS double differences calculated from the GPS signals. It is observed that the two sets of GPS double differences obtained agree with each other most of the times but doesn't agree few times when there is huge cloud covering the reference signal.

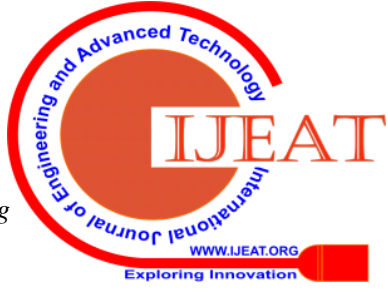


Also it is observed that when the reference satellite is covered with huge cloud or rain a large error is seen in the GPS signal. To get accurate predictions the system time of weather radar is matched with the GPS time because for the weather radar precise timing is not necessary as it doesn't matter if it starts raining one minute earlier or one minute late but the same is not the case with the GPS because the satellite position changes by large extent even in single minute. Also it is observed that weather front moving towards a site also induce large delay in the GPS signal.

From the analysis performed in Chapter 5, it was found that the modeling of troposphere delays requires surface conditions in terms of temperature, humidity, and pressure, as well as the temperature and humidity along the signal propagation path. For the calculation of troposphere propagation delay difference over a $5 \mathrm{~km}$ baseline, the pressure information is less important than the temperature and humidity. Based on the results presented in Chapter 5, it can be concluded that weather radar data by itself is not sufficient to accurately estimate the troposphere delay in real time. However, the weather radar data has been instrumental in showing that the contributions from N3 and N4 on the differential troposphere delay are less significant than those introduced by $\mathrm{N} 2$. Troposphere delay due to the combined effect of $\mathrm{N} 3$ and $\mathrm{N} 4$, representing clouds, rain, snow and hail, was less than $5 \%$ of the delay introduced by $\mathrm{N} 2$ representing water vapor during a severe weather event. This is an important result as it supports the threat model documented in [2]. The radar reflectivity values provide good estimates of rainfall and clouds, but they are not well correlated with humidity or temperature. Existing radar model equations were used to estimate the relative humidity along the signal propagation path for reflectivity values between 45 and $70 \mathrm{dBz}$ [17], but these were not found to accurately represent the actual atmospheric conditions. The results in Chapter 5 also show that the weather radar data can be used as an indicator of significant troposphere delay differences between two locations.

Future research is recommended to determine the sensitivity of the troposphere delay due to temperature and relative humidity along the signal propagation path. This study should provide the accuracy of the temperature and relative humidity measurements needed to determine the troposphere delay. Next, research is recommended in the area of improved humidity and temperature profile measurements using dual frequency band radar measurements as documented. concluded that after mitigation of PUI, the performance of the proposed cognitive relay network is significantly improved. for your paper size.

\section{REFERENCES}

1. Steven R. Strom. "Charting a Course Toward Global Navigation". The Aerospace Corporation. Retrieved on 2008-06-27.

2. http://www.abbreviations.com/NAVSTAR

3. History of GPS", usinfo.state.gov (February 3, 2006).

4. HowStuffWorks: How GPS Receivers WorkAccessed May 14, 2006.

5. globalsecurity.org [1].

6. P. Daly. "Navstar GPS and GLONASS: global satellite navigation systems". IEEE.

7. Dana, Peter H. GPS Orbital Planes. August 8, 1996.

8. What the Global Positioning System Tells Us about Relativity. Accessed January 2, 2007.

9. GPS Overview from the NAVSTAR Joint Program Office. Accessed December 15, 2006
10. Massatt, Paul and Brady, Wayne. "Optimizing performance through constellation management", Crosslink, Summer 2002, pages 17-21.

11. USNO NAVSTAR Global Positioning System. Accessed May 14, 2006.

12. Note that though there are many receiver manufacurers, they almost all use one of the chipsets produced for this purpose. An example: "GPS Receiver Chip Performance Survey". GPS Technology Reviews.

13. N. Nithiyanandam, K. Venkatesh, M. Rajesh, Transfer The Levels Of The Monitored Carbon, Nitrogen Gases From The Industries, International Journal of Recent Technology and Engineering, Volume-7 Issue-6S3 April, 2019.

14. Sivanesh Kumar, A., Brittoraj, S., Rajesh, M., Implementation of RFID with internet of things, Journal of Recent Technology and Engineering, Volume-7 Issue-6S3 April, 2019.

15. Rajesh, M., Sairam, R., Big data and health care system using mlearningJournal of Recent Technology and Engineering, Volume-7 Issue-6S3 April, 2019.

16. Rajesh, M., and J. M. Gnanasekar. "Path Observation Based Physical Routing Protocol for Wireless Ad Hoc Networks." Wireless Personal Communications 97.1 (2017): 1267-1289. 